\title{
Endothelial cell survival during angiogenesis requires the pro-survival protein MCL1
}

\author{
EC Watson ${ }^{1,2}$, L Whitehead ${ }^{2,3}$, RH Adams ${ }^{4,5}$, G Dewson $^{2,6}$ and L Coultas ${ }^{\star, 1,2}$
}

\begin{abstract}
Angiogenesis is essential to match the size of blood vessel networks to the metabolic demands of growing tissues. While many genes and pathways necessary for regulating angiogenesis have been identified, those responsible for endothelial cell (EC) survival during angiogenesis remain largely unknown. We have investigated the in vivo role of myeloid cell leukemia 1 (MCL1), a pro-survival member of the BCL2 family, in EC survival during angiogenesis. EC-specific deletion of Mcl1 resulted in a dosedependent increase in EC apoptosis in the angiogenic vasculature and a corresponding decline in vessel density. Our results suggest this apoptosis was independent of the BH3-only protein BIM. Despite the known link between apoptosis and blood vessel regression, this was not the cause of reduced vessel density observed in the absence of endothelial MCL1. Rather, the reduction in vessel density was linked to ectopic apoptosis in regions of the angiogenic vasculature where EC proliferation and new vessel growth occurs. We have therefore identified MCL1 as an essential survival factor for ECs that is required for blood vessel production during angiogenesis.
\end{abstract}

Cell Death and Differentiation (2016) 23, 1371-1379; doi:10.1038/cdd.2016.20; published online 4 March 2016

Endothelial cell (EC) development begins shortly after gastrulation with the production of precursor angioblasts that differentiate into $\mathrm{EC}$ and coalesce to form the initial vessels of the embryo. ${ }^{1}$ These vessels are subsequently remodeled and expanded by angiogenesis: the coordinated proliferation, migration, differentiation and remodeling of ECs and blood vessels. ${ }^{2}$ Inappropriate activation of angiogenesis in adults is associated with a range of diseases, including diabetic retinopathy, retinopathy of prematurity, wet age-related macular degeneration and cancer. ${ }^{3}$ Angiogenic ECs are sensitive to apoptosis. In the retina this occurs as part of normal development during vessel remodeling, ${ }^{4}$ and in response to hyperoxic stress, ${ }^{5}$ while vascular endothelial growth factor $A$ (VEGF-A) inhibition can cause EC apoptosis in the case of tumor angiogenesis. ${ }^{6}$ Despite the susceptibility of angiogenic ECs to apoptosis, the proteins responsible for their survival remain poorly understood.

Angiogenesis involves the sprouting of new vessels from existing ones. This requires the specification of endothelial 'tip' cells that initiate new vessel sprouts, and endothelial 'stalk' cells, that trail the tip cell and proliferate, supplying the growing sprout with new ECs. ${ }^{7}$ Sprouting angiogenesis lays down an immature, largely uniform vessel plexus that is subsequently remodeled into a refined, efficient conduit for blood flow. Such remodeling requires the selective removal of certain vessel segments. Sprouting occurs at the periphery of the expanding vessel network (the sprouting zone), whereas remodeling takes place behind the sprouting zone, in the vessels laid down earlier (the remodeling zone). EC proliferation predominantly occurs in the sprouting zone, with some occurring in the remodeling zone around veins, whereas very little occurs near remodeling arteries. ${ }^{8,9}$ In contrast, apoptosis has been reported to associate with remodeling arteries. ${ }^{10}$ Thus, EC proliferation and apoptosis in the angiogenic network appear largely compartmentally separated.

The BCL2 family controls the cell 'intrinsic' pathway of apoptotic cell death. It consists of both pro-survival and proapoptotic members. Apoptotic stimuli activate one or more of the so-called BH3-only BCL2 proteins (BIM, BID, BAD, BIK, HRK, PUMA and noxa), which then activate the effector proapoptotic BCL2 proteins (BAK, BAX and possibly BOK), either through direct binding, or indirectly, by inactivating the prosurvival family members (BCL2, MCL1, BCLW, BCLXL and A1). ${ }^{11}$ Once unleashed, BAK and BAX cause the release of apoptogenic factors, such as cytochrome $c$, from mitochondria. This triggers the activation of caspases, intracellular proteases that demolish the cell. The intrinsic apoptosis pathway is indispensible for physiologically relevant EC apoptosis as mice lacking both of the apoptotic effector proteins BAK and BAX contain persistent hyaloid vasculature, a network that normally undergoes apoptotic regression due to EC apoptosis. ${ }^{12}$ The BH3-only protein BIM is central to EC apoptosis as it is required for hyaloid vessel regression, ${ }^{4,13}$ EC apoptosis during normal retina angiogenesis, ${ }^{4,13}$

\footnotetext{
${ }^{1}$ Development and Cancer Division, The Walter and Eliza Hall Institute of Medical Research, 1G Royal Parade, Parkville, VIC, Australia; ${ }^{2}$ Department of Medical Biology, University of Melbourne, 1G Royal Parade, VIC, Australia; ${ }^{3}$ Systems Biology and Personalised Medicine Division, The Walter and Eliza Hall Institute of Medical Research, $1 G$ Royal Parade, Parkville, VIC, Australia; ${ }^{4}$ Department of Tissue Morphogenesis, Max Planck Institute for Molecular Biomedicine, Roentgenstrasse 20, Münster D, Germany; ${ }^{5}$ Faculty of Medicine, University of Münster, Münster D, Germany and ${ }^{6}$ Cell Signalling and Cell Death Division, The Walter and Eliza Hall Institute of Medical Research, 1G Royal Parade, Parkville, VIC, Australia

*Corresponding author: L Coultas, Development and Cancer Division, The Walter and Eliza Hall Institute of Medical Research, 1G Royal Parade, Parkville 3052, VIC, Australia; Tel: +61 39345 2860; Fax: +61 39347 0852; E-mail: Icoultas@ @ehi.edu.au

Abbreviations: DPBS, Dulbecco's phosphate buffered saline solution; E, embryonic day; EC, endothelial cell; hCD4, human cluster of differentiation 4); MCL1, myeloid cell leukemia 1; P, postnatal day; PECAM1, platelet/endothelial cell adhesion molecule 1; TKO, triple knockout; VEGF-A, vascular endothelial growth factor A Received 18.3.15; revised 07.1.16; accepted 29.1.16; Edited by G Melino; published online 04.3.16
} 
vaso-obliteration of immature retinal vessels in response to hyperoxia ${ }^{13}$ and apoptosis of tumor-associated ECs following VEGF-A blockade. ${ }^{6}$ While it is clear the BCL2-mediated apoptosis pathway is responsible for EC death, little is known about which pro-survival proteins of this pathway normally promote EC survival through apoptosis suppression. Here, we demonstrate that the pro-survival protein MCL1 is essential for the survival of ECs undergoing angiogenesis in vivo, particularly those ECs located in the proliferative regions of the angiogenic network.

\section{Results}

MCL1 is essential for EC development. Germline loss of Mcl1 results in peri-implantation lethality. ${ }^{14}$ To investigate the role of MCL1 in EC development, we deleted $\mathrm{Mcl} 1$ by crossing a Tie2-cre transgene (which is active in ECs and their progenitors from embryonic day (E)7.5 (ref. 15)), with a conditional $M c / 1$ allele in which an human cluster of differentiation 4 (hCD4) reporter is expressed from the Mcl1 locus on cre-mediated excision. ${ }^{16}$ These mice will hereafter be referred to as $M c / 1^{E C}$. Flow cytometric analysis of hCD4 reporter expression in the embryo proper and yolk sac of E10.5 embryos showed a dose-dependent shift in hCD4 expression on ECs from $\mathrm{Mcl} / 1^{\mathrm{EC} /+}$ and $M c / 1^{E C / E C}$ embryos, demonstrating efficient deletion of the $\mathrm{Mcl} 1$ allele (Figure 1a). The Mcl1 ${ }^{E C / E C}$ embryos were present at the expected Mendelian ratios at E10.5 (Table 1), however, fewer mutants were observed at E14.5, with the majority of those that were present showing one or a combination of edema, hemorrhage or presumed blood-filled lymphatics (Figure 1b,Table 1). Several $M c / 1^{E C / E C}$ embryos at E14.5 lacked heartbeat or were undergoing resorption. This indicated embryonic lethality was occurring at or prior to E14.5.

$M c / 1^{E C / E C}$ mutants were significantly under-represented at weaning $\left(P=0.001, X^{2}\right.$-test) (Table 1$)$, however, a small number did survive to adulthood. While hCD4 expression was present on ECs from the kidney, lung and skeletal muscle in adult $M c / 1^{E C /+}$ mice, hCD4 was not detected on ECs from the few surviving $M c / 11^{E C / E C}$ mice (Figure 1c), and the deleted $(\triangle) \mathrm{Mcl} 1$ allele was extremely low or undetectable by genomic PCR in ECs sorted from those few adult $M c / 1^{E C / E C}$ mice $(n=3)$ (Figure 1d). This demonstrates that ECs in the surviving $M c / 1^{E C / E C}$ mice had escaped cre-mediated Mcl1 deletion. Consistent with this, we noted that hCD4 expression in ECs at E10.5 was bimodal in approximately one in seven embryos (combined $\mathrm{Mcl} 1^{\mathrm{EC} /+}$ and $M c / 1^{E C / E C}$ embryos) (Figure 1e). Given hCD4 expression was observed uniformly in ECs from four out of five $\mathrm{MCl} 1^{E C / E C}$ embryos at E10.5, bimodal presentation of hCD4 was not likely a result of EC death due to reduced MCL1, but was more likely due to cre inefficiency prior to E10.5. Such cre inefficiency in $M c / 1^{E C / E C}$ mice would result in a proportion of ECs in which $\mathrm{Mcl} / 1$ was not deleted, enabling them to out-compete Mcl1-deficient cells and permit formation of a normal vasculature and survival into adulthood.

Embryonic angiogenesis is disrupted in the absence of MCL1. To determine the effect of MCL1 loss on angiogenesis, we investigated the development of the subcutaneous vasculature in dorsal skin in embryos at E14.5. Blood vessels emerge laterally then migrate toward the dorsal midline to fully cover the dorsal skin between E14.5 and E15.5. ${ }^{17}$ Given the variability of $\mathrm{Mcl} 1$ deletion we observed in the $M c / 1{ }^{E C / E C}$ embryos, we analyzed only those embryos with evidence of edema as a means to select embryos with the highest likelihood of efficient cre-mediated excision of the Mcl1 allele. Vascularization of the dorsal skin was delayed in $M c / 1{ }^{E C / E C}$ embryos, with an increased avascular area present at the midline of E14.5 embryos (Figure 1f). Furthermore, vessel sprouts at the leading edge of the growing vasculature in $M c / 1^{E C / E C}$ embryos were elongated, with fewer side branches (Figure 1g). Attempts to quantify EC apoptosis were complicated by an inability to clearly determine whether apoptotic cells were endothelial or hematopoietic cells (in which Tie2-cre is also active ${ }^{15}$ ) and excessive autofluorescence from circulating and extravasated erythrocytes (due to hemorrhage). These data indicate that angiogenesis was abnormal in embryos lacking endothelial MCL1, however, to what extent this was due to a cell-autonomous survival defect or a response to stretching of the dorsal skin by the underlying edema was unclear.

MCL1 is a dose-dependent survival factor for angiogenic endothelium. To investigate the consequences of $\mathrm{Mcl} / \mathrm{loss}$ on EC survival in greater detail, we crossed the $\mathrm{Mcl} 1$ conditional allele with the tamoxifen-inducible, EC-specific Cdh5(PAC)-creERT2 transgene ${ }^{18}$ and induced $M c / 1$ deletion in neonates. These mice are hereafter referred to as $M c / 1^{i E C}$ mice. ECs of the kidney and skeletal muscle from postnatal day $(P) 5$ pups showed efficient cre-mediated deletion of the Mcl1 allele following tamoxifen administration based on hCD4 expression (Figure 2a). McliEC mice showed no expression of the hCD4 on CD45 ${ }^{+}$Ter119- white blood cells in the bone marrow, confirming the specificity of the inducible cre for ECs and not hematopoietic cells (Figure 2a). To determine the effect of inducible $\mathrm{Mcl} 1$ deletion on angiogenesis, we examined retinal vasculature in $M c / 1^{i E C / I E C}$ pups following tamoxifen administration. Quantification of the retinal vascular density at P5 revealed a gene-dosage-dependent effect of $\mathrm{Mcl1}$ loss on vascular density, with $\mathrm{Mcl} \mathrm{1}^{\mathrm{iEC} / \mathrm{EEC}}$ retinas containing significantly less vessel surface area, vessel segments and vessel branch points than controls (Figures $2 \mathrm{~b}$ and $\mathrm{c}$ ). Given MCL1 is a pro-survival protein, EC apoptosis was assessed at P5 by staining for platelet/ endothelial cell adhesion molecule 1 (PECAM1), collagen IV and cleaved (activated) caspase-3 (Figure 3a). Apoptotic ECs were quantified across the whole vasculature of mutant and control retinas by confocal imaging. $\mathrm{Mcl} / 1^{i E C /+}$ and $M c / 1^{i E C / i E C}$ pups contained significantly more apoptotic ECs than controls (Figure $3 b$ ), and demonstrated a gene-dosagedependent effect on EC survival.

Reduced vessel density in Mcl1 mutants is not due to increased blood vessel regression. We examined whether the reduction in vasculature in MCL1 mutants was caused by increased vessel regression, a common consequence of EC apoptosis. ${ }^{5,10,19,20}$ We quantified vessel regression in mutant and control retinas based on the presence of collagen IV sleeves devoid of PECAM1 signal, which mark sites of vessel 
a

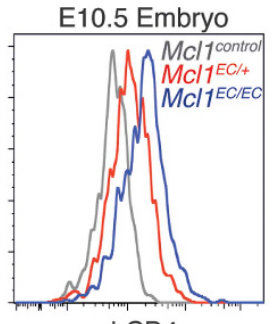

hCD4

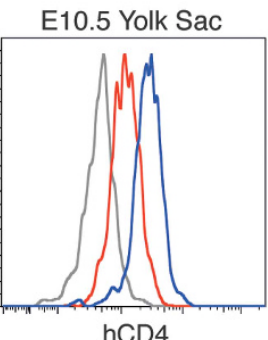

hCD4

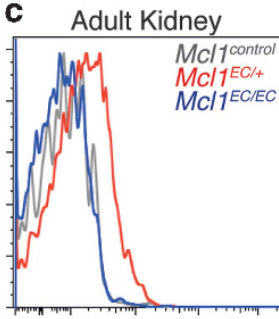

hCD4

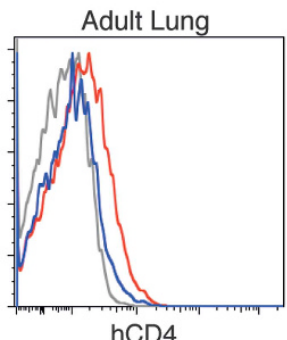

hCD4 b Control

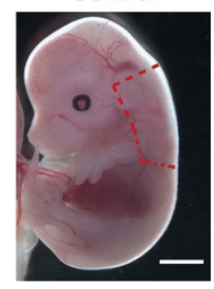

MCI1ECIEC

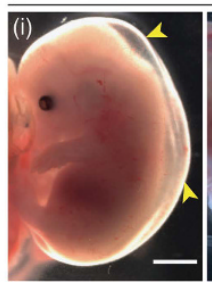

d

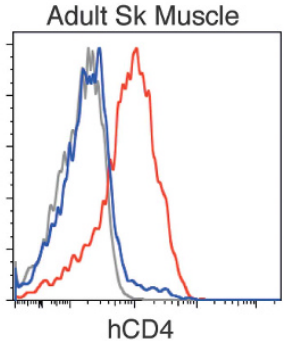

e

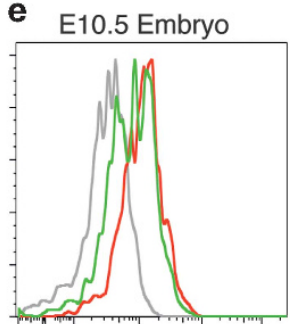

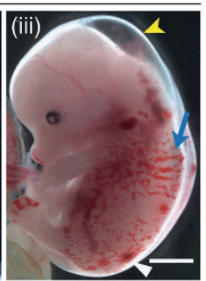

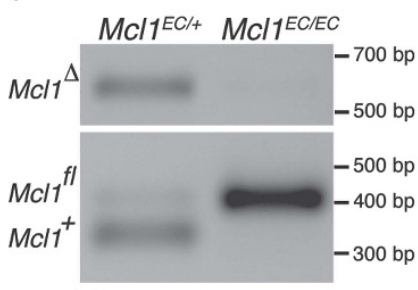

hCD4
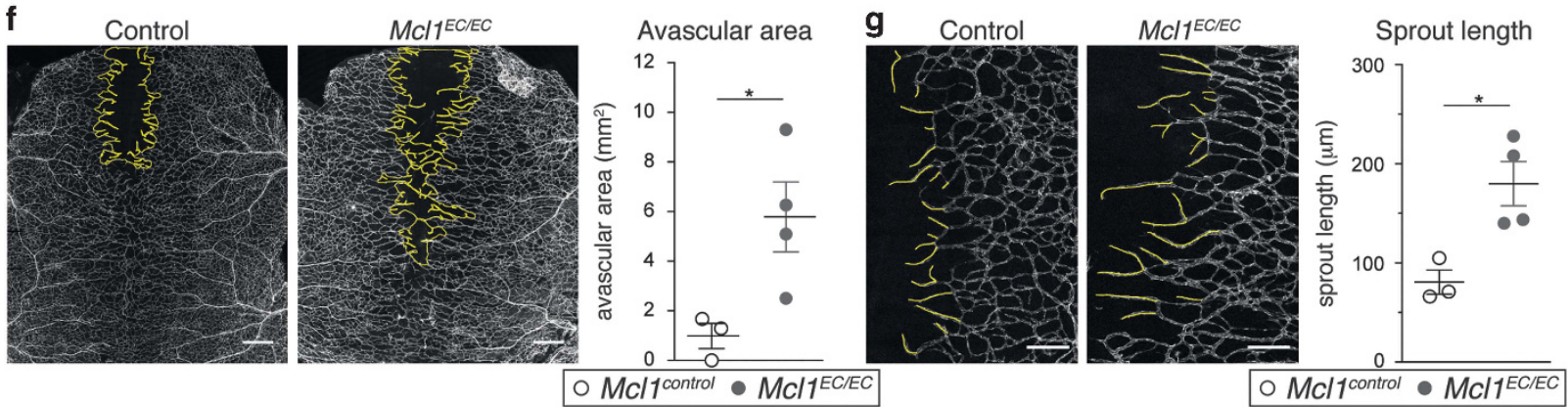

Figure $1 \mathrm{MCl} 1^{E C / E C}$ embryos show a range of phenotypes at E14.5 while surviving adult $M c / 1^{E C / E C}$ mice do not show deletion of $M c / 1$ in ECs. (a) Representative flow cytometric analysis of hCD4 expression (a reporter of $M c / 1^{f l}$ allele recombination) on E10.5 embryo proper and yolk sac ECs from Mcl $1^{\text {control }}\left(n=12\right.$, gray lines), Mcl $1^{E C /+}$

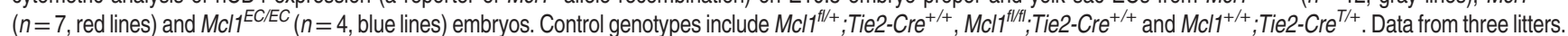
(b) Representative images of $M c / 1^{\text {control }}(n=60)$ and $M C / 1^{E C / E C}(n=18)$ E14.5 embryos. Data from 18 litters. Yellow arrowhead indicates edema, white arrowhead indicates hemorrhage and blue arrow indicates possible blood-filled lymphatic vessels. Red line demarcates region of dorsal skin used for quantification of vasculature in $F$ and $G$. Scale $\mathrm{bar}=2 \mathrm{~mm}$. (c) Representative flow cytometric analysis of $\mathrm{hCD} 4$ expression on adult kidney, lung and skeletal muscle ECs from $M c / 1^{\text {control }}\left(n=6\right.$, gray line), Mcl $1^{E C /+}(n=6$, red

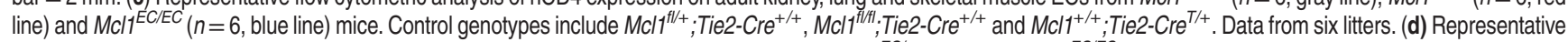
genomic PCR result from fluorescence-activated cell sorting sorted kidney ECs isolated from $M C / 1^{E C /+}(n=3)$ and $M c / 1^{E C / E C}(n=3)$ mice. (e) Flow cytometric analysis of hCD4 expression from an E10.5 Mcli $1^{i E C /+}$ embryo $\left(n=1\right.$, green line) showing inefficient recombination of the conditional allele compared with $M c / 1^{\text {control }}$ ( $n=12$, gray line) and an $M c / 1^{E C /+}$ embryo with efficient Mcl1 allele deletion ( $n=7$, red line). (f) Representative images of E14.5 dorsal skin vasculature stained for PECAM1 from Mcl $1^{\text {control }}(n=3)$ and $\operatorname{Mc} 11^{E C / E C}(n=4)$ embryos. Yellow line demarcates avascular region. Scale bar $=500 \mu \mathrm{m}$. Graph shows quantification of avascular region from $M c / 1^{\text {control }}(n=3$, open circles) and $M C / 1^{E C / E C}\left(n=4\right.$, closed circles) embryos. (g) Representative images of dorsal skin vasculature quantified for sprout length stained for PECAM1 from Mcl $7^{\text {Control }}(n=3)$ and $M C / 1^{E C / E C}(n=4)$. Yellow lines trace sprouts to nearest branch point. Scale bar $=200 \mu \mathrm{m}$. Graph shows quantification of mean sprout length per embryo from Mcl1 ${ }^{\text {control }}(n=3$, open circles) and $M C / 1^{E C I E C}\left(n=4\right.$, closed circles) embryos. Mean \pm S.E.M. ${ }^{*} P \leqslant 0.05$, Student's two-tailed $t$-test

regression ${ }^{21,22}$ (Figure $3 c$ ). Vessel regression was quantified in the region of the remodeling vein and in the sprouting region as these were sites of significantly increased apoptosis in the mutants (see below), but we saw no difference in the amount of vessel regression in either location irrespective of genotype (Figures $3 d$ and e). Increased vessel regression was therefore unlikely to explain the reduced vasculature observed in the MCL1 mutants.

MCL1 prevents EC apoptosis in proliferative and sprouting regions of the angiogenic vessel network. Having excluded vessel regression, we investigated whether the distribution of apoptotic ECs could account for the reduced vascularization of mutant retinas. We quantified the number of apoptotic ECs within the sprouting zone (area external to red circles in Figure 4a), and their proximity to maturing arteries and veins within the remodeling zone (area within red circles in Figure 4a). Apoptotic ECs in P5 control retinas were predominantly located around the developing arteries, with few seen in the venous or sprouting territories (Figures $4 \mathrm{a}$ and b). Relative to controls, EC apoptosis in mutant retinas was only marginally increased (1.5-fold) around arteries, which was not significant. In contrast, EC apoptosis in both $M c / 1^{i E C /+}$ and $M c / 1^{i E C / i E C}$ retinas was significantly increased around veins in the remodeling zone (Figures $4 a$ and $b$ ) and in the sprouting zone of $M C / 1^{i E C / i E C}$ mice, regions in which EC proliferation and new vessel sprouting occurs. ${ }^{8,9}$ Notably, homozygote mutants had significantly more apoptotic cells 
Table 1 Observed and expected numbers of controls and mutant $M c / 1^{\mathrm{EC}}$ by age

\begin{tabular}{|c|c|c|c|c|}
\hline & Control & $M c l 1^{\mathrm{EC} /+}$ & $M c / 1^{\mathrm{EC} / \mathrm{EC}}$ & TOTAL \\
\hline \multicolumn{5}{|l|}{$E 10.5^{a}$} \\
\hline Observed & 12 & 8 & 5 & 25 \\
\hline Expected & 15 & 6 & 4 & 25 \\
\hline \multicolumn{5}{|l|}{$E 14.5^{b}$} \\
\hline Observed & 60 & 35 & $18(2)^{c}$ & 110 \\
\hline Expected & 55 & 27.5 & 27.5 & 110 \\
\hline \multicolumn{5}{|l|}{ Weaning $^{d}$} \\
\hline Observed & 96 & 37 & $3^{e}$ & 136 \\
\hline Expected & 85 & 34 & 17 & 136 \\
\hline
\end{tabular}

antercrosses between $\mathrm{Mcl}^{\mathrm{fl} /+}$ or $\mathrm{Mcl}^{\mathrm{fl} / \mathrm{fll}}$ females and $\mathrm{Mcl}^{\mathrm{fl} /+} ; \mathrm{Tie}_{2}-\mathrm{Cre}^{\mathrm{T} /+}$

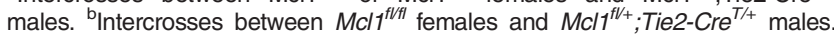
${ }^{c}$ Number in parenthesis denotes embryos that were phenotypically normal. ${ }^{d}$ Intercrosses between $\mathrm{Mcl} / 1^{f /+}$ females and $\mathrm{Mcl} 1^{f /+} ;$ Tie2-Cre $^{T /+}$ males. ${ }^{e} \mathrm{P}=0.001$, $x^{2}$-test

than heterozygotes in both these areas. These results demonstrate that MCL1 is required for the survival of ECs located in those regions of the vasculature necessary for new vessel production.

$\mathrm{BIM}$ is not required for $\mathrm{EC}$ apoptosis or reduced vascular density caused by the loss of MCL1. In addition to its prosurvival role, MCL1 has been show to regulate mitochondrial fusion, cristae morphology and ATP generation through oxidative phosphorylation. ${ }^{23}$ We therefore sought to determine the extent to which the ectopic EC apoptosis observed in the $\mathrm{Mcl} 1$ mutants was responsible for the angiogenic phenotype. Given BIM is responsible for retina EC apoptosis in vivo, ${ }^{4,13}$ we investigated whether its loss would be sufficient to rescue the $M c / 1$ phenotype. $M c / 1^{i E C / i E C}$ mice were crossed to $\mathrm{Bim}^{-/-}$mice to generate $\mathrm{Mcl} / 1^{i E C / i E C} \mathrm{Bim}^{-1-}$ double mutants. While the number of double mutants analyzed was small, we could find no evidence that vascular density in $\mathrm{Mcl1}^{\text {iEC/iEC }} \mathrm{Bim}^{-/-}$double mutant retinas was changed relative to $M c / 1^{i E C / i E C}$ mice (Figures $5 a$ and b). Similarly, EC apoptosis in the small number of $M c / 1^{i E C / i E C} \mathrm{Bim}^{-1-}$ double mutant retinas analyzed was indistinguishable from $M c / 1^{i E C / i E C}$ mice (Figure $5 \mathrm{c}$ ). These results suggest that $E C$ apoptosis following the loss of MCL1 proceeds independently of BIM.

Simultaneous deletion of Bak and Bax rescues the EC apoptosis defect, embryonic lethality and angiogenesis defects in Tie2-cre-deleted Mcl1 mutants. As deletion of Bim was insufficient to rescue the phenotype of the $\mathrm{Mcl} / 1$ mutants, we generated mice in which both of the intrinsic apoptosis effectors, Bak and Bax, were deleted specifically in the endothelium using Tie2-cre. Triple mutant mice lacking Mcl1, Bak and Bax in ECs (hereafter referred to as Mcl1 ${ }^{\text {TKO/TKO; }}$ triple knockout (TKO)) were born at the expected Mendelian frequency (expected: 4, observed: 4) and survived to at least 6 weeks of age with no overt phenotype $(n=2)$. In contrast to $M c / 1^{E C / E C}$ mice, where no EC deletion of the Mcl1 allele was observed (Figure 1c), ECs of the kidney, lung and skeletal muscle from postnatal $\mathrm{Mcl1}{ }^{\text {TKO} / T K O}$ mice showed efficient cre-mediated deletion of
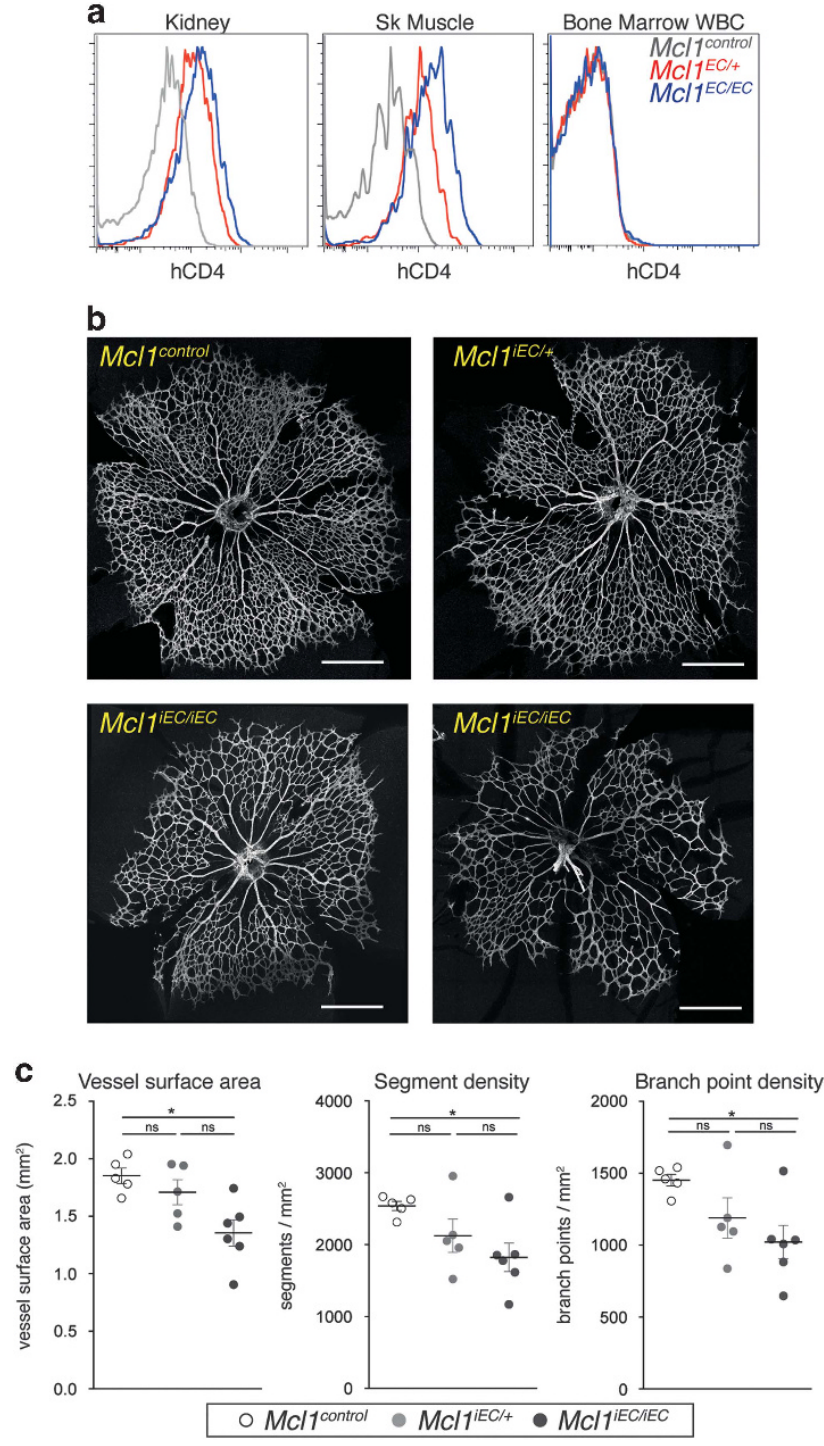

Figure $2 \mathrm{Mcl} 1^{\mathrm{iEC} / \mathrm{EEC}}$ mice show reduced retinal vasculature at $\mathrm{P} 5$. (a) Representative flow cytometric analysis of hCD4 expression on P5 kidney ECs, skeletal muscle ECs and bone marrow white blood cells (WBC). EC data are from $M c / 1^{\text {control }}\left(n=10\right.$, gray lines), McliEC/+ $\left(n=13\right.$, red lines) and Mcl1 ${ }^{i E C / i E C}(n=10$, blue lines) pups from seven litters. WBC data are from $M c / 1^{\text {control }}(n=4$, gray line), $M c / 1^{i E C /+}\left(n=3\right.$, red line) and $M c / 1^{i E C / E C}$ ( $n=6$, blue line) pups from three litters. Control genotypes in each population include $\mathrm{Mcl}^{f / /+} ; \mathrm{Cdh5}(\mathrm{PAC})$-creERT2 ${ }^{+/+}$, $M c / 1^{f l / f l}$; Cdh5(PAC)-creERT2 ${ }^{+/+}$and $M c l 1^{+/+} ; \operatorname{Cdh5}(P A C)-c r e E R T 2^{T /+}$. (b) Representative images of whole retinal vasculature stained for collagen IV from Mcl $1^{\text {control }}$ $(n=5), \mathrm{Mc}^{j \mathrm{iEC} /+}(n=5)$ and two $\mathrm{Mc}^{\text {iEC/iEC }}(n=6)$ retinas at P5. Data from six litters. Scale bar $=500 \mu \mathrm{m}$. (c) Vessel surface area (left panel), vessel segment density (center panel) and branch point density (right panel) in retinas from $\mathrm{Mcl} /{ }^{\text {control }}$ ( $n=5$, open circles), Mcl $1^{i E C /+}\left(n=5\right.$, gray circles) and $M c / 1^{i E C / i E C}(n=6$, closed circles) pups at P5. Vessel surface area was measured based on collagen $\mathrm{IV}^{+}$signal. Branch point density and segment density were quantified on skeletonized collagen IV signal and normalized to total area from two fields of view per retina. Data from six litters. Mean \pm S.E.M. Not significant (NS): $P>0.05 ;{ }^{*} P \leqslant 0.05$, one-way ANOVA with Tukey's post hoc multiple comparison test

the Mcl1 allele based on hCD4 expression (Figure 6a). Analysis of retinal vasculature at P5 showed that the amount of vasculature in $M c / 1^{\text {TKO/TKO }}$ pups was normal (Figures $6 \mathrm{~b}$ and c) and that EC apoptosis was completely absent 
a

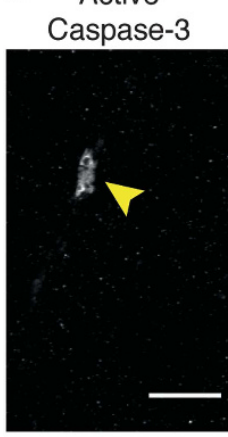

C
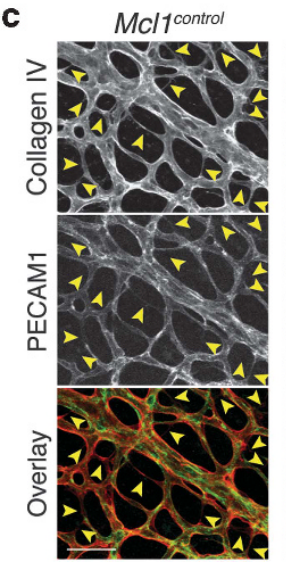

MCl1'iEC/iEC

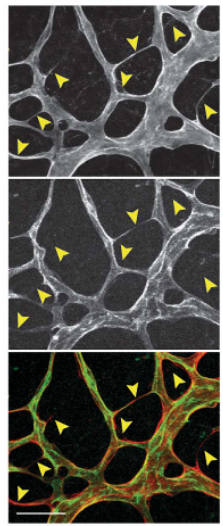

Collagen IV

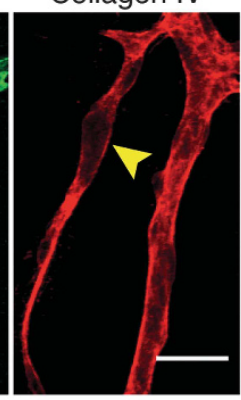

d

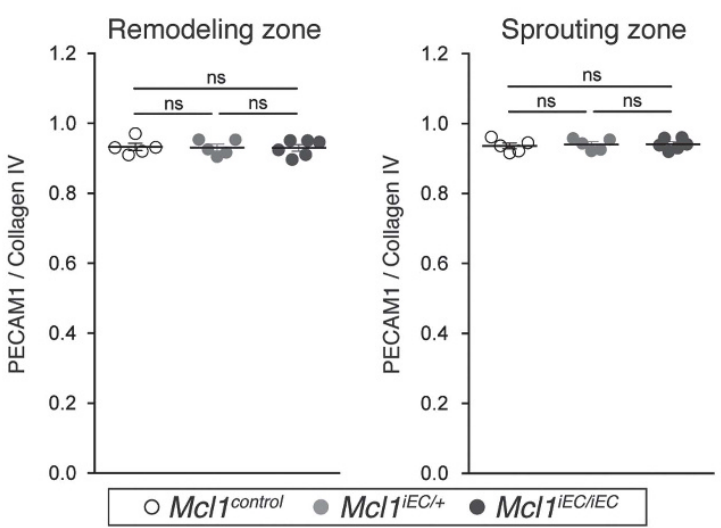

b

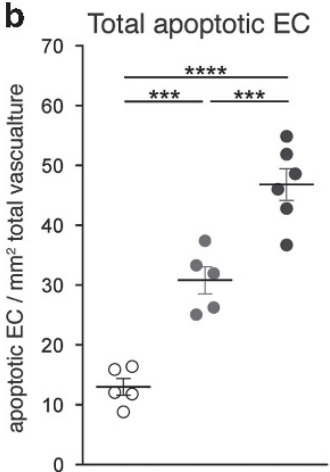

Figure 3 Deletion of $M c / 1$ results in increased EC apoptosis in Mcli ${ }^{\text {EEC/EC }}$ retinas at P5. (a) Representative image of apoptotic retina EC. Yellow arrowhead indicates apoptotic EC. PECAM1 (green), Collagen IV (red), active caspase 3 (gray). Scale bar $=30 \mu \mathrm{m}$. (b) Total apoptotic ECs per retina relative to vascular surface area. (c) Representative examples of regressing vessels in $M c / 1^{\text {control }}$ and $M C / 1^{i E C / E C}$ retinas. Yellow arrowheads indicate regressing vessels. Overlay shows collagen IV (red) and PECAM1 (green). Scale bar $=50 \mu \mathrm{m}$. (d and e) Vessel regression, determined by the ratio of PECAM1-positive vessel segments to collagen IV-positive vessel segments in the venous remodeling zone (d) and in the sprouting zone (e). All data are presented as mean \pm S.E.M. from $M c / 1^{\text {control }}(n=5$, open circles), McliEC/+ $(n=5$, gray circles) and $M c / 1^{i E C / i E C}$ ( $n=6$, closed circles) retinas. Data from six litters. Not significant (NS): $P>0.05 ;{ }^{* \star \star} P \leqslant 0.001 ;{ }^{\star \star \star \star} P \leqslant 0.0001$, one-way ANOVA with Tukey's post hoc multiple

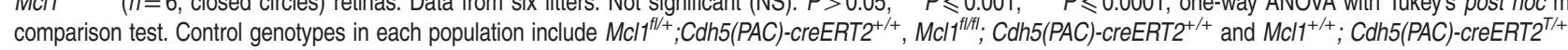

(Figure 6c). While the number of triple mutants analyzed at P5 was small $(n=2)$, these data strongly suggest that the Mcl1 angiogenesis phenotype is due to compromised EC survival.

\section{Discussion}

EC apoptosis is dependent on members of the BCL2 pathway. While the requirement for pro-apoptotic pathway members is well-defined, the identity of those that promote EC survival in vivo is largely unknown. Here we have shown that MCL1 is essential for EC survival during angiogenesis in vivo.

The loss of endothelial MCL1 during embryonic stages resulted in hemorrhage, edema and embryonic lethality. However, given the Tie2-cre transgene used to delete Mcl1 also causes deletion in hematopoietic cells ${ }^{15}$ and MCL1 is required for the survival of hematopoietic stem cells, ${ }^{24}$ it seems likely that hematopoietic defects will contribute to the embryonic lethality of $M c / 1^{E C / E C}$ mutants, making the EC-specific role of MCL1 in the survival of these animals difficult to discern. Our use of the inducible Cdh5(PAC)creERT2 transgene, which we showed was not active in hematopoietic cells in neonates, confirms that MCL1 is essential for EC survival in a cell-autonomous manner during postnatal angiogenesis.

Our results show that during angiogenesis, MCL1 is a key survival factor for ECs in the proliferative regions of the angiogenic vasculature, and its role in angiogenesis is to promote new vessel production rather than to prevent inappropriate vessel regression. It is not possible to conclude from our data whether MCL1 is required for the survival of ECs that are actively proliferating, or whether its loss simply limits the number of cells available to proliferate and generate new vessels. Several stimuli have been reported to promote EC apoptosis through downregulation of MCL1 in vitro. These include hypoxia, ${ }^{25,26}$ suppression of $\mathrm{ERK}^{27}$ and Rho inhibition. ${ }^{28}$ Conversely, the S1P receptor agonist FTY720P was reported to delay apoptosis in serum-starved HUVEC by upregulating MCL1 ${ }^{29}$ How MCL1 activity is directed to the proliferative regions of the angiogenic network in vivo will be an important question to address.

$\mathrm{BIM}$ is required for $\mathrm{EC}$ apoptosis in the angiogenic retina, ${ }^{4,13}$ and forms endogenous complexes with MCL1 in ECs. ${ }^{27}$ However, Bim deletion was not sufficient to rescue the apoptosis defect caused by the loss of $\mathrm{Mcl} / 1$ in the limited number of mice studied here. While the small number of 

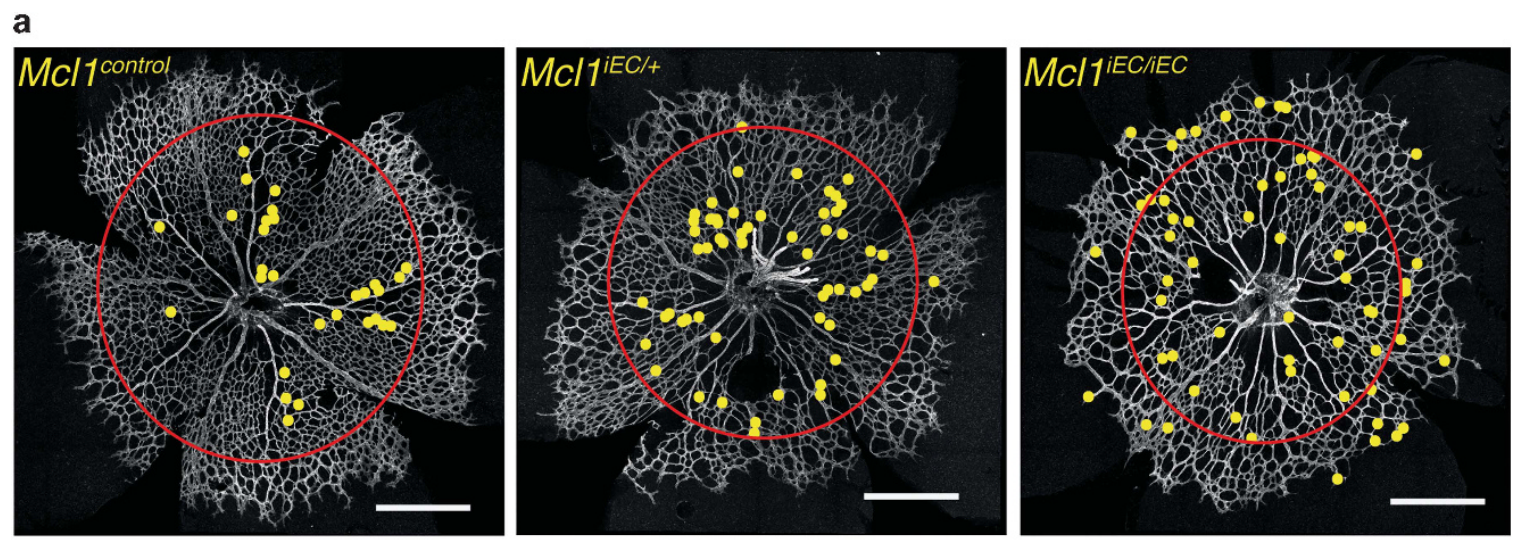

b

b Artery apoptotic EC
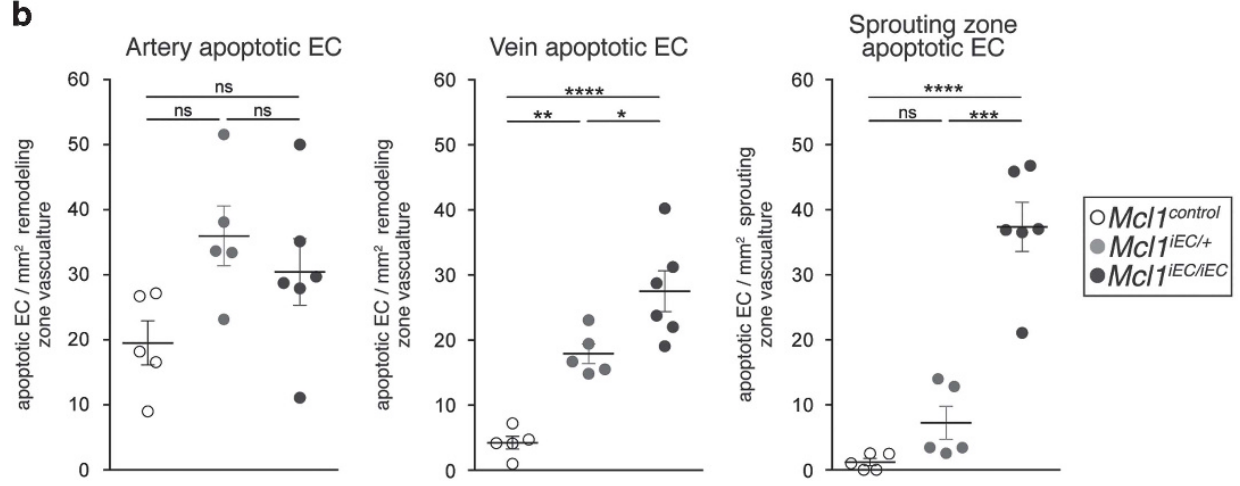

0

Figure 4 MCL1 is required for region-specific EC survival during angiogenesis. (a) Representative images from P5 Mc/1 ${ }^{\text {control }}, M C / 1^{\text {iEC/ }}$ and $M C / 1^{\text {iEC/IEC }}$ retinas stained for collagen IV. Yellow dots indicate the position of apoptotic ECs, Red circle demarcates the boundary between remodeling (inside) and sprouting (outside) regions. Scale bar $=500 \mu \mathrm{m}$. (b) Quantification of apoptotic EC distribution normalized to vascular surface area by region (remodeling or sprouting) in P5 retinas. Apoptotic ECs within the remodeling region were assigned to either arterial or venous regions based on the identity of the closest major vessel. All data are presented as mean \pm S.E.M. from Mcl $1^{\text {control }}$ ( $n=5$, open circles), Mcl $1^{i E C /+}\left(n=5\right.$, gray circles) and Mcl1 ${ }^{i E C / i E C}\left(n=6\right.$, closed circles) retinas. Data from six litters. Not significant (NS): $P>0.05 ;{ }^{*} P \leqslant 0.05 ;{ }^{\star *} P \leqslant 0.01$; ${ }^{* * \star} P \leqslant 0.001 ;{ }^{* * \star} P \leqslant 0.0001$, one-way ANOVA with Tukey's post hoc multiple comparison test. Control genotypes in each population include $M c / 1^{f / /+} ; C d h 5(P A C)-c r e E R T 2^{+/+}$,

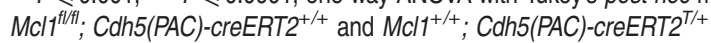

double mutant animals analyzed prevents conclusive interpretation, the data suggest that BIM is not essential for EC apoptosis to proceed following loss of MCL1, and that BIM is not the major antagonist of MCL1 in this system. This result is consistent with findings in the hematopoietic system, where PUMA, not BIM, is the dominant antagonist of MCL $1 .{ }^{30}$ In ECs however, PUMA has been reported to promote EC survival during angiogenesis. ${ }^{31}$ MCL1 also binds the BH3-only proteins BID and noxa, ${ }^{11}$ but the roles of these proteins in promoting EC apoptosis following MCL1 loss is yet to be determined. However, simultaneous deletion of both Bak and Bax was sufficient to overcome the $M c / 1$ vascular phenotype. While the number of mice analyzed was small and prevent conclusive interpretation, the data strongly suggest that the requirement for MCL1 in angiogenic ECs is primarily due to its pro-survival role, rather than a defect in oxidative phosphorylation as reported in other cell types. ${ }^{23,32}$ This is consistent with findings that ECs rely predominantly on glycolysis for energy production, and that this further increases in response to pro-angiogenic signals such as VEGF-A. ${ }^{33}$

Our analysis of the distribution of apoptotic ECs in nonmutant control mice confirms the results of others that suggest EC apoptosis is associated with arterial vessel pruning. ${ }^{10}$ However, our observations in the Mcl1 mutant mice show that
EC apoptosis does not always cause vessel regression, as has been suggested from other mutant phenotypes. ${ }^{10,34,35}$ This raises the question of whether EC apoptosis is functionally required for vessel pruning or is simply a consequence of it. Indeed, studies in zebrafish have shown that while EC apoptosis is associated with vessel pruning, it is largely dispensable for this process, with EC rearrangements being the primary driver of vessel pruning. ${ }^{36,37}$ Recent detailed analysis of the angiogenic retinal vasculature in mice found that only $\sim 5 \%$ of regressing vessels found during normal angiogenesis actually contained apoptotic ECs. ${ }^{38}$ Whether EC apoptosis is functionally necessary for vessel pruning in the mouse retina is yet to be determined.

Of the pro-survival BCL2 proteins, only BCL2 itself has previously been investigated for its function in EC survival in vivo, where it was also implicated in EC survival during retina angiogenesis. ${ }^{39}$ However, its role and regulation may be quite distinct to that of MCL1: whereas we found MCL1 was important for EC survival in the proliferative regions of the vasculature at $\mathrm{P} 5$, apoptosis in $\mathrm{Bcl}^{-/}$retinas was reported at $\mathrm{P} 14,{ }^{39}$ when very little EC proliferation is occurring. ${ }^{9}$ It seems highly likely that pro-survival proteins will have distinct roles within in the vasculature to promote its survival and health. Our findings have clearly demonstrated that MCL1 has a 

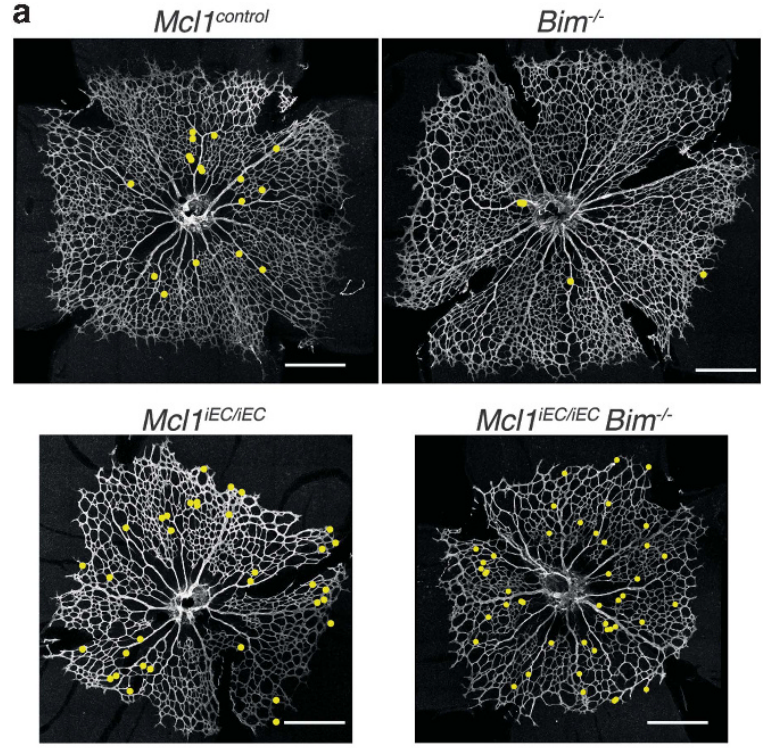

b

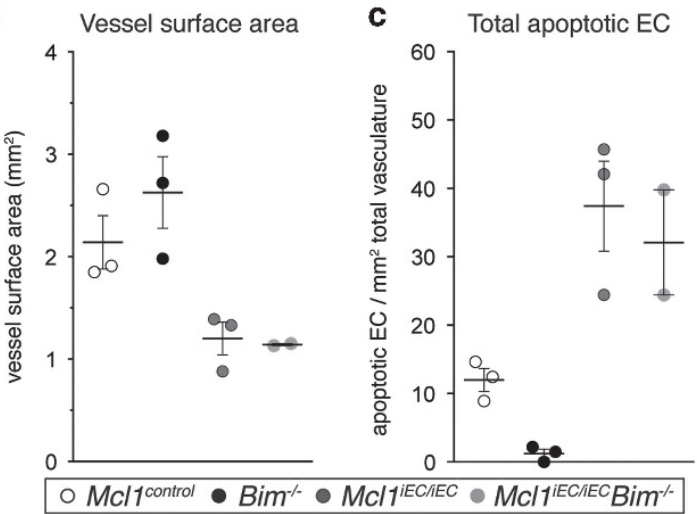

Figure 5 Loss of BIM does not prevent EC apoptosis or restore vascular density in MCl1 ${ }^{\text {EEC/EC }}$ mutants. (a) Representative images from P5 $\mathrm{MCl}^{\text {control }}, \mathrm{Bim}^{-1-}$, $M C / 1^{i E C / i E C}$ and $M C / 1^{i E C / i E C}$ Bim $^{-1-}$ retinas stained for collagen IV. Yellow dots indicate the position of apoptotic ECs. Scale bar $=500 \mu \mathrm{m}$. (b) Vessel surface area and (c) total apoptotic ECs per retina relative to vascular surface area in retinas from Mcl $1^{\text {control }}\left(n=3\right.$, open circles), Bim ${ }^{-1-}\left(n=3\right.$, black circles), Mcl $1^{i E C / I E C}(n=3$, dark gray circles) and $M c / 1^{i E C / / E C} \mathrm{Bim}^{-/-}$pups at P5 ( $n=2$, light gray circles). Data from six litters. Control genotypes in each population include $\mathrm{Mcl1}^{\mathrm{fl} /} ; \mathrm{Cdh} 5(\mathrm{PAC})$ -

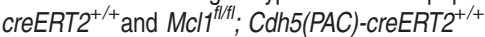

non-redundant role to promote EC survival during proliferative blood vessel network expansion.

\section{Materials and Methods}

Mice. All experiments involving animals were performed with procedures approved by the Walter \& Eliza Hall Institute of Medical Research Animal Ethics Committee. Conditional Mcl1 mice, ${ }^{16}$ Bim mice, ${ }^{40}$ Conditional Bax mice, ${ }^{41}$ Bak null mice, ${ }^{42}$ Cdh5(PAC)-creERT2 mice ${ }^{18}$ and Tie2-cre mice ${ }^{15}$ have all been described previously. Animals were maintained on an inbred C57BL/6 background. Noon on the day a vaginal mating plug was observed was termed E0.5. The day of birth was termed P0. Tamoxifen $(50 \mu \mathrm{g} / \mathrm{dose}$ in sterile corn oil) was administered via intragastric injection to litters on P2 or P2 and P3.

Immunohistochemical analysis of whole mount embryonic dorsal skin vasculature. E14.5 embryo proper were fixed in $4 \%$ paraformaldehyde for $2 \mathrm{~h}$ at $4{ }^{\circ} \mathrm{C}$. Dorsal skin tissue was dissected and extra a
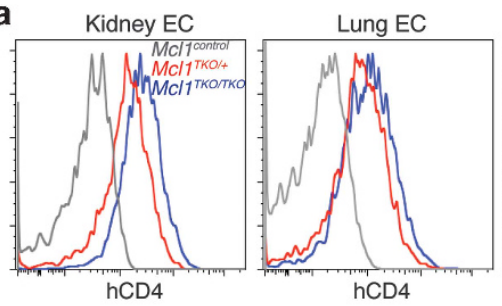

Sk Muscle EC

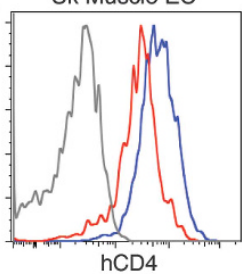

b
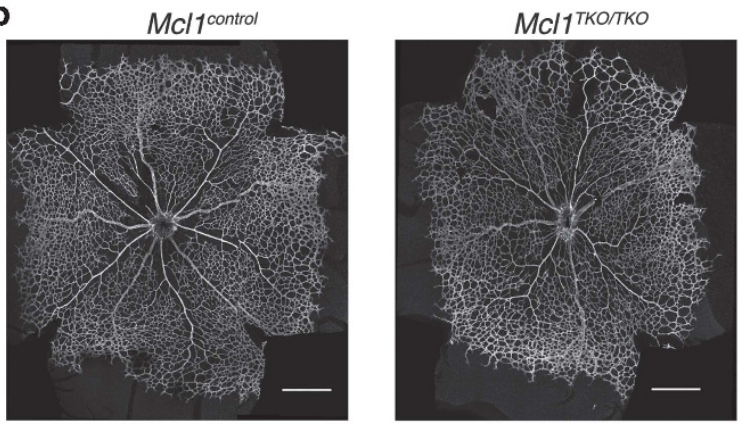

c

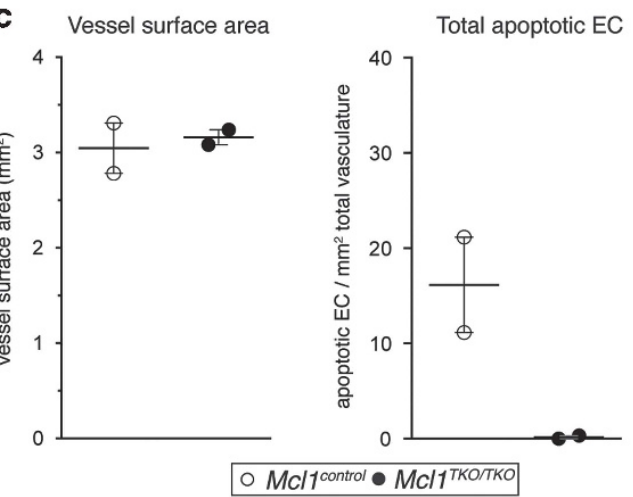

Figure 6 Simultaneous deletion of Bak and Bax rescues the EC apoptosis defect, embryonic lethality and angiogenesis defect Mcl1 mutants. (a) Representative flow cytometric analysis of hCD4 expression on kidney ECs, lung ECs and skeletal muscle ECs. Data are from postnatal Mcl $1^{\text {control }}\left(n=3\right.$, gray lines), $\mathrm{Mcl}^{\mathrm{TKO} /+}(n=4$,

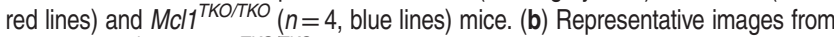
P5 Mcl1 ${ }^{\text {control }}$ and $M \mathrm{Mcl}^{\text {TKOITKO }}$ retinas stained for collagen IV. Scale bar $=500 \mu \mathrm{m}$. (c) Vessel surface area and total apoptotic ECs per retina relative to vascular surface area in retinas from $M c / 1^{\text {control }}$ ( $n=2$, open circles) and $M c / 1^{\text {TKOTTKO }}(n=2$, black circles) pups at P5 layers of tissue and muscle removed. Dorsal skin samples were washed through 25 , $50,75 \%$ and stored in $100 \%$ methanol at $-20^{\circ} \mathrm{C}$ at least overnight until further processing. Samples were washed through a reverse methanol series prior to staining with rabbit anti-collagen IV antibody $(20 \mu \mathrm{g} / \mathrm{ml}$, AB756P, Millipore, Kilsyth, VIC, Australia) and hamster anti-PECAM1 $(5 \mu \mathrm{g} / \mathrm{ml}$, clone 2H8, ab78739, Abcam, Cambridge, United Kingdom) in $2 \%$ normal donkey serum, $1 \%$ Triton X-100 in Dulbecco's phosphate buffered saline solution (DPBS) overnight at $4{ }^{\circ} \mathrm{C}$. Samples were washed $(0.01 \%$, Triton X-100 in DPBS) then incubated with secondary antibodies: Donkey anti-rabbit IgG DyLight 488 (711-485-152, Jackson ImmunoResearch, West Grove, PA, USA) and Goat anti-hamster IgG Alexa-Fluor 647 (127$605-160$, Jackson ImmunoResearch) in blocking buffer overnight at $4{ }^{\circ} \mathrm{C}$. Samples were then washed before being mounted on slides in ProLong Gold antifade reagent (Invitrogen, Mulgrave, VIC, Australia) and imaged using a Leica SP8 (Leica, North Ryde, NSW, Australia) confocal microscope with a $\times 10 / 0.40$ NA objective using Leica Application Suite software (Leica, North Ryde, NSW, Australia) or a Zeiss LSM 780 (Zeiss, North Ryde, NSW, Australia) confocal microscope with a × 10/0.45 NA objective using Zen software (Zeiss, North Ryde, NSW, Australia). 
Quantification of avascular space was performed using Adobe Photoshop for manual tracing of avascular area followed by quantification of area using FIJ distribution of ImageJ software (NIH, Bethesda, MD, USA) from confocal z-stack projections of dorsal skin vasculature. ${ }^{43}$ Sprout length in dorsal skin vasculature was measured to the nearest branch point manually using FIJI distribution of ImageJ software on a $1500 \mu \mathrm{m}$ length of sprouting front.

Immunohistochemical analysis of retinal vasculature. Eyes were enucleated and fixed in $4 \%$ paraformaldehyde for $2 \mathrm{~h}$ at $4{ }^{\circ} \mathrm{C}$. Retinas were then dissected, incubated in blocking buffer (2\% normal donkey serum, $1 \%$ Triton X-100 in DPBS) then stained with rat anti-PECAM1 $(5 \mu \mathrm{g} / \mathrm{ml}$, clone MEC 13.3, 553370, BD Pharmingen), goat anti-collagen IV antibody ( $14 \mu \mathrm{g} / \mathrm{ml}, \mathrm{AB} 769$, Millipore) and rabbit anti-cleaved (activated) caspase-3 antibody (used at 1/50, clone 5A1, 9664, Cell Signaling Technologies, Beverly, MA, USA) in blocking buffer overnight at $4^{\circ} \mathrm{C}$. Retinas were washed $(0.01 \%$ Triton $X-100$ in DPBS) then incubated with secondary antibodies: anti-rat IgG DyLight 488 (711-485-152, Jackson ImmunoResearch), antigoat IgG Alexa-Fluor 647 (705-605-147, Jackson ImmunoResearch) and anti-rabbit IgG Cy3 (711-165-152, Jackson ImmunoResearch) in blocking buffer overnight at $4{ }^{\circ} \mathrm{C}$. Retinas were then washed before being mounted on slides in ProLong Gold antifade reagent. All retina imaging was performed using a Leica SP8 confocal microscope with a $\times 20 / 0.75$ NA objective using Leica Application Suite software. Apoptotic ECs were defined as cleaved caspase- $3^{+} /$PECAM1 ${ }^{+}$cells enclosed by collagen IV in the $x-, y$ - and $z$-axis from confocal $z$-stack images. Quantification of cleaved caspase-3-positive ECs was performed manually using FIJI distribution of ImageJ software from confocal $z$ stacks of entire retinas. Classification of an EC as apoptotic required agreement by two investigators. Vessel surface area measurements of whole retinas were quantified using FIJI software based on the collagen IV signal in maximum intensity projection images generated from confocal $z$ stacks of entire retinas. Vasculature at the optic nerve head and residual hyaloid vasculature were manually masked and excluded from vessel surface area quantification. Quantification was performed using the thresholding function and included removal of non-specific background using the despeckle filter. Quantification of vessel area in whole retinas using this method was verified using independent methodology in a semi-automated manner through a custom written FIJI macro, by an independent investigator who was blinded to genotype. The steps of the macro involved initial processing and filtering, specifically a maximum intensity projection of collagen IV signal and background subtraction, followed by manual outlining of optic nerve head and residual hyaloid vasculature to exclude these from the measurements. The macro then segmented the retina using the triangle method of automatic thresholding. Vessel segment density and branch point density were quantified across two fields of view/retina using the Angiogenesis Tube Formation module of Metamorph image processing software (version 7.8.2.0, Molecular Devices, Sunnyvale, CA, USA) on the collagen IV signal. Vessel regression was determined as a ratio of $\mathrm{PECAM} 1^{+}$vessel segment length to collagen $\mathrm{IV}^{+}$vessel segment length. Vessel regression analysis was performed as follows: binary masks of PECAM1 and collagen IV channels were produced through auto thresholding and the use of various image and morphological filters. A collagen $\mathrm{IV}^{+}$PECAM1 ${ }^{-}$vessel segment mask (regressing vessels) was generated by subtracting the PECAM1 channel mask from the collagen IV channel mask. Collagen $\mathrm{IV}^{+} \mathrm{PECAM}^{-}$vessel segment mask and the collagen IV channel mask were then skeletonized and their total vessel lengths measured. These steps were all implemented in a custom FIJI macro allowing for full automation of the data set. Data were compiled from two to three fields of view/retina from the remodeling vein region and four to six fields of view/retina from the sprouting region. Vessel regression analysis was undertaken by investigators who were blinded to genotype.

Flow cytometry analysis and sorting of ECs and blood cells. Whole E10.5 embryos were dissected free of extra-embryonic membranes. Yolk sac and embryo proper were separately digested in DPBS containing $0.25 \mathrm{WU} / \mathrm{ml}$ Liberase TM (Roche), $10 \mu \mathrm{g} / \mathrm{ml}$ DNase I (Roche, Dee Why, NSW, Australia) and $5 \mathrm{mM} \mathrm{MgCl} 2$ for $40 \mathrm{~min}$ at $37^{\circ} \mathrm{C}$ with constant agitation. Dissociated cells were stained with anti-PECAM1-APC (clone MEC 13.3, 551262, BD Pharmingen, North Ryde, NSW, Australia) anti-ICAM2-FITC (clone 3C4(mIC2/4), 557444, BD Biosciences, North Ryde, NSW, Australia), anti-hCD4-PE (clone RPA-T4, 555347, BD Pharmingen) and anti-CD41-PEcy7 (MWReg30, 25-0411-82, eBiosciences, San Diego, CA, USA). Cells were analyzed using a BD LSR II flow cytometer (BD Bioscience) and ECs defined as PECAM1 ${ }^{\mathrm{Hi}}$ ICAM $^{\mathrm{Hi}} \mathrm{CD} 1^{-}$cells. Dead cells were excluded based on DAPI uptake. Tissues from neonates were digested as described above and passed through $50-\mu \mathrm{m}$ filter mesh. Dissociated cells were stained using anti-PECAM1-APC (clone 390, 17-0311, eBiosciences), anti-ICAM2FITC, anti-CD45.2-PerCPcy5.5 (clone 104, 561096, BD Pharmingen) and antihCD4-PE. ECs were defined as PECAM1 ${ }^{\mathrm{Hi}}$ ICAM2 ${ }^{\mathrm{Hi}} \mathrm{CD} 45^{-}$. Dead cells were excluded based on DAPI uptake. For white blood cell analysis, bone marrow was flushed from one femur of each mouse with balanced salt solution containing $1 \%$ heat-inactivated fetal calf serum and dissociated by trituration using a $21 \mathrm{G}$ needle (BD, North Ryde, NSW, Australia). Dissociated cells were stained with anti-Ter119APC (prepared in-house), anti-CD45.2-PerCPcy5.5 and anti-hCD4-PE. White blood cells were defined as Ter119- CD45.2 $2^{+}$. Dead cells were excluded based on DAPI uptake. Flow cytometric analysis of adult mice was as described for neonates, but using anti-PECAM1-APC clone MEC 13.3 (BD Pharmingen). Adult kidney ECs for fluorescence-activated cell sorting sorting were prepared as described above for flow cytometric analysis and sorted using an Aria llu sorter (BD bioscience). ECs were defined as PECAM1 ${ }^{\mathrm{Hi}} \mathrm{CD}^{-} 5^{-}$. In some cases, anti-ICAM2-FITC (clone 3C4(mIC2/4), 557444, BD Biosciences) or anti-CD105-PE (MJ7/18, 12-1051, eBioscience) were used as additional EC markers. Dead cells were excluded based on DAPI uptake. For flow cytometric analysis, ECs were defined as PECAM1 $1^{\mathrm{Hi}}$ ICAM2 $2^{\mathrm{Hi}} \mathrm{CD} 45^{-}$. White blood cells from bone marrow were defined as Ter119CD $45.2^{+}$. Dead cells were excluded based on DAPI uptake. All flow cytometric analysis data were acquired using an LSR II flow cytometer and analyzed using FlowJo software. ECs were sorted using an Aria llu sorter.

Genomic PCR analysis. Genomic PCR was performed on DNA extracted from sorted kidney ECs. The primers used were: Mcl1-5': $5^{\prime}$-gcacaatccgtccgcgagccaa-3', Mcl1-3': 5'-gccgcagtacaggttcaag-3' and Mcl1-del: 5'-gagtcagcgcgatcattcagct-3'. Expected product sizes were: 327 bp (WT allele), 409 bp (fl allele) and 567 bp (del allele).

Statistics. Statistical analysis was performed using one-way analysis of variance test with Tukey's multiple comparison test unless otherwise indicated. Two-tailed $\chi^{2}$-tests were used to analyze offspring frequency.

\section{Conflict of Interest}

The authors declare no conflict of interest.

Acknowledgements. We thank M Scott and $K$ Rogers for expert imaging assistance, E Trounson and MN Koenig for expert technical assistance, E Lanera, $G$ Dabrowski and D Cooper for expert animal care and procedures, Douglas Green (St Jude Children's Research Hospital, Memphis) for Bax conditional allele mice, Masashi Yanagisawa (UT Southwestern) for Tie2-cre mice, A Strasser and P Bouillet (the Walter and Eliza Hall Institute) for generously providing Mcl1 conditional mice, $\mathrm{Bim}^{-1-}$ mice and for informative discussion, RCA Symons (the Royal Melbourne Hospital), AK Voss (the Walter and Eliza Hall Institute) and members of the Development and Cancer Division (the Walter and Eliza Hall Institute) for informative discussion. This work was made possible through Victorian State Government Operational Infrastructure Support and Australian Government NHMRC IRIISS. This work was supported by the National Health and Medical Research Council, Australia (Project Grant: 1010638). LC and GD are supported by Australian Research Council Future Fellowships (LC: 110100891. GD: 100100791). LC is supported by the LEW Carty Charitable Fund.

\section{Author contributions}

ECW performed research, analyzed data and wrote the paper. LW generated analytical tools and analyzed data. RHA provided essential reagents. GD analyzed data. LC designed research, analyzed data and wrote the paper.

1. Coultas L, Chawengsaksophak K, Rossant J. Endothelial cells and VEGF in vascular development. Nature 2005; 438: 937-945.

2. Potente $M$, Gerhardt $H$, Carmeliet $P$. Basic and therapeutic aspects of angiogenesis. Cell 2011; 146: 873-887.

3. Carmeliet P. Angiogenesis in life, disease and medicine. Nature 2005; 438: 932-936.

4. Koenig MN, Naik E, Rohrbeck L, Herold MJ, Trounson E, Bouillet P et al. Pro-apoptotic BIM is an essential initiator of physiological endothelial cell death independent of regulation by FOXO3. Cell Death Differ 2014; 21: 1687-1695.

5. Alon T, Hemo I, Itin A, Pe'er J, Stone J, Keshet E. Vascular endothelial growth factor acts as a survival factor for newly formed retinal vessels and has implications for retinopathy of prematurity. Nat Med 1995; 1: 1024-1028. 
6. Naik E, O'Reilly LA, Asselin-Labat ML, Merino D, Lin A, Cook M et al. Destruction of tumor vasculature and abated tumor growth upon VEGF blockade is driven by proapoptotic protein Bim in endothelial cells. J Exp Med 2011; 208: 1351-1358.

7. Phng LK, Gerhardt H. Angiogenesis: a team effort coordinated by notch. Dev Cell 2009; 16 : 196-208.

8. Gerhardt H, Golding M, Fruttiger M, Ruhrberg C, Lundkvist A, Abramsson A et al. VEGF guides angiogenic sprouting utilizing endothelial tip cell filopodia. J Cell Biol 2003; 161 : 1163-1177.

9. Ehling M, Adams S, Benedito R, Adams RH. Notch controls retinal blood vessel maturation and quiescence. Development 2013; 140: 3051-3061.

10. Simonavicius N, Ashenden M, van Weverwijk A, Lax S, Huso DL, Buckley CD et al. Pericytes promote selective vessel regression to regulate vascular patterning. Blood 2012; 120: 1516-1527.

11. Czabotar PE, Lessene G, Strasser A, Adams JM. Control of apoptosis by the BCL-2 protein family: implications for physiology and therapy. Nat Rev Mol Cell Biol 2014; 15: 49-63.

12. Hahn $\mathrm{P}$, Lindsten T, Tolentino M, Thompson CB, Bennett J, Dunaief JL. Persistent fetal ocular vasculature in mice deficient in bax and bak. Arch Ophthalmol 2005; 123: 797-802.

13. Wang S, Park S, Fei P, Sorenson CM. Bim is responsible for the inherent sensitivity of the developing retinal vasculature to hyperoxia. Dev Biol 2011; 349: 296-309.

14. Rinkenberger JL, Horning S, Klocke B, Roth K, Korsmeyer SJ. Mcl-1 deficiency results in peri-implantation embryonic lethality. Genes Dev 2000; 14: 23-27.

15. Kisanuki YY, Hammer RE, Miyazaki J, Williams SC, Richardson JA, Yanagisawa M. Tie2-Cre transgenic mice: a new model for endothelial cell-lineage analysis in vivo. Dev Biol 2001; 230: 230-242.

16. Vikstrom I, Carotta S, Luthje K, Peperzak V, Jost PJ, Glaser S et al. Mcl-1 is essential for germinal center formation and B cell memory. Science 2010; 330: 1095-1099.

17. James JM, Nalbandian A, Mukouyama YS. TGFbeta signaling is required for sprouting lymphangiogenesis during lymphatic network development in the skin. Development 2013; 140: $3903-3914$

18. Wang Y, Nakayama M, Pitulescu ME, Schmidt TS, Bochenek ML, Sakakibara A et al. Ephrin-B2 controls VEGF-induced angiogenesis and lymphangiogenesis. Nature 2010; 465 483-486.

19. Baffert F, Le T, Sennino B, Thurston G, Kuo CJ, Hu-Lowe D et al. Cellular changes in norma blood capillaries undergoing regression after inhibition of VEGF signaling. Am J Physiol Heart Circ Physiol 2006; 290: H547-H559.

20. Meeson A, Palmer M, Calfon M, Lang R. A relationship between apoptosis and flow during programmed capillary regression is revealed by vital analysis. Development 1996; 122 : 3929-3938.

21. Inai $T$, Mancuso $M$, Hashizume $H$, Baffert $F$, Haskell A, Baluk $P$ et al. Inhibition of vascular endothelial growth factor (VEGF) signaling in cancer causes loss of endothelial fenestrations, regression of tumor vessels, and appearance of basement membrane ghosts. Am J Pathol 2004; 165: 35-52

22. Phng LK, Potente M, Leslie JD, Babbage J, Nyqvist D, Lobov I et al. Nrarp coordinates endothelial Notch and Wnt signaling to control vessel density in angiogenesis. Dev Cell 2009; 16: 70-82.

23. Perciavalle RM, Stewart DP, Koss B, Lynch J, Milasta S, Bathina M et al. Anti-apoptotic MCL-1 localizes to the mitochondrial matrix and couples mitochondrial fusion to respiration. Nat Cell Biol 2012; 14: 575-583.

24. Opferman JT, Iwasaki H, Ong CC, Suh $\mathrm{H}$, Mizuno S, Akashi K et al. Obligate role of antiapoptotic MCL-1 in the survival of hematopoietic stem cells. Science 2005; 307: 1101-1104.
25. Li C, Issa R, Kumar P, Hampson IN, Lopez-Novoa JM, Bernabeu C et al. CD105 prevents apoptosis in hypoxic endothelial cells. J Cell Sci 2003; 116: 2677-2685.

26. Kucharzewska $P$, Welch JE, Svensson KJ, Belting M. The polyamines regulate endothelia cell survival during hypoxic stress through PI3K/AKT and MCL-1. Biochem Biophys Res Commun 2009; 380: 413-418.

27. Sun HL, Tsai AC, Pan SL, Ding Q, Yamaguchi H, Lin CN et al. EPOX inhibits angiogenesis by degradation of Mcl-1 through ERK inactivation. Clin Cancer Res 2009; 15: 4904-4914.

28. Hippenstiel S, Schmeck B, N'Guessan PD, Seybold J, Krull M, Preissner K et al. Rho protein inactivation induced apoptosis of cultured human endothelial cells. Am J Physiol Lung Cell Mol Physiol 2002; 283: L830-L838.

29. Rutherford C, Childs S, Ohotski J, McGlynn L, Riddick M, MacFarlane S et al. Regulation of cell survival by sphingosine-1-phosphate receptor S1P1 via reciprocal ERK-dependent suppression of Bim and PI-3-kinase/protein kinase C-mediated upregulation of Mcl-1. Cell Death Dis 2013; 4: e927.

30. Delbridge AR, Opferman JT, Grabow S, Strasser A. Antagonism between MCL-1 and PUMA governs stem/progenitor cell survival during hematopoietic recovery from stress. Blood 2015; 125: 3273-3280.

31. Zhang F, Li Y, Tang Z, Kumar A, Lee $C$, Zhang $L$ et al. Proliferative and survival effects of PUMA promote angiogenesis. Cell reports 2012; 2: 1272-1285.

32. Wang X, Bathina M, Lynch J, Koss B, Calabrese C, Frase S et al. Deletion of MCL-1 causes lethal cardiac failure and mitochondrial dysfunction. Genes Dev 2013; 27: 1351-1364.

33. De Bock K, Georgiadou M, Carmeliet P. Role of endothelial cell metabolism in vesse sprouting. Cell Metab 2013; 18: 634-647.

34. Korn C, Scholz B, Hu J, Srivastava K, Wojtarowicz J, Arnsperger T et al. Endothelial cellderived non-canonical Wnt ligands control vascular pruning in angiogenesis. Development 2014; 141: 1757-1766.

35. Savant S, La Porta S, Budnik A, Busch K, Hu J, Tisch N et al. The orphan receptor Tie1 controls angiogenesis and vascular remodeling by differentially regulating Tie2 in tip and stalk cells. Cell Rep 2015; 12: 1761-1773.

36. Kochhan E, Lenard A, Ellertsdottir E, Herwig L, Affolter M, Belting HG et al. Blood flow changes coincide with cellular rearrangements during blood vessel pruning in zebrafish embryos. PLoS One 2013; 8: e75060.

37. Chen Q, Jiang L, Li C, Hu D, Bu JW, Cai D et al. Haemodynamics-driven developmental pruning of brain vasculature in zebrafish. PLoS Biol 2012; 10: e1001374.

38. Franco CA, Jones ML, Bernabeu MO, Geudens I, Mathivet T, Rosa A et al. Dynamic endothelial cell rearrangements drive developmental vessel regression. PLoS Biol 2015; 13 : e1002125.

39. Wang S, Sorenson CM, Sheibani N. Attenuation of retinal vascular development and neovascularization during oxygen-induced ischemic retinopathy in $\mathrm{Bcl}_{-2}{ }^{-/-}$mice. Dev Biol 2005; 279: 205-219.

40. Bouillet $\mathrm{P}$, Cory S, Zhang LC, Strasser A, Adams JM. Degenerative disorders caused by Bcl-2 deficiency prevented by loss of its BH3-only antagonist Bim. Dev Cell 2001; 1 : 645-653.

41. Knudson CM, Tung KS, Tourtellotte WG, Brown GA, Korsmeyer SJ. Bax-deficient mice with lymphoid hyperplasia and male germ cell death. Science 1995; 270: 96-99.

42. Lindsten T, Ross AJ, King A, Zong WX, Rathmell JC, Shiels HA et al. The combined functions of proapoptotic Bcl-2 family members bak and bax are essential for normal development of multiple tissues. Mol Cell 2000; 6: 1389-1399.

43. Schindelin J, Arganda-Carreras I, Frise E, Kaynig V, Longair M, Pietzsch T et al. Fiji: an open-source platform for biological-image analysis. Nat Methods 2012; 9: 676-682. 